\title{
Sistema de gestión de calidad y su impacto en la productividad del sector textil.
}

Quality management system and its impact on the productivity of the textile sector.

Ramos Viteri Eufemia Alejandrina. ${ }^{1}$, Velastegui López Luis Efrain. ${ }^{2}$, Tatiana Carrasco Ruano. ${ }^{3}$ \& Cepeda Cahuatijo Evelyn Lizeth. ${ }^{4}$

DOI: https://doi.org/10.33262/visionariodigital.v2i1.34

\section{Resumen.}

El sistema de gestión de calidad beneficia a toda la organización porque influye directamente en la reducción de costos operativos e interviene de manera sinérgica en lineamientos para dirigir y controlar los objetivos de la cultura en calidad que vinculada con la productividad ofrece mayores oportunidades al sector textil.

La productividad inicia con eficiencia y ésta busca mayor rendimiento con mínimo de recursos. En la productividad también está la efectividad cuyo propósito es conseguir los objetivos propuestos. La eficiencia y la efectividad dan como resultado la eficacia que es la base de la productividad.

Utilizamos la encuesta como instrumento de la investigación descriptiva y aplicamos la fórmula matemática de la productividad: el sistema productivo sobre la cantidad de recursos utilizados se concluye que las empresas textiles con certificación ISO 9001 tienen un índice de 1,44 en y productividad y las que no poseen el resultado es de 1,20. La productividad total de la industria manufacturera ecuatoriana que consta en el CIIU3, numeral 17 del Instituto Nacional de Estadísticas y Censo proporciona el índice estándar de 2,0 dólares por unidad de insumo; por tanto, se requiere socializar la certificación internacional para que el sector textil incremente el índice de productividad.

Palabras clave: Sistema, Gestión de calidad, Productividad, Textil.

\footnotetext{
${ }^{1}$ Docente, Universidad Técnica de Ambato, Ambato, Ecuador, eufemiaaramos@uta.edu.ec

${ }^{2}$ Docente, Universidad Técnica de Ambato, Ambato, Ecuador, le.velastegui@uta.edu.ec

${ }^{3}$ Universidad Internacional de La Rioja UNIR, España, tcarrascor@yahoo.cpm

${ }^{4}$ Estudiante, Universidad Técnica de Ambato, Ambato, Ecuador, evelynlizethcepeda@gmail.com 


\section{Abstract.}

The quality management system benefits the entire organization because it influences directly in the reduction of operating costs and intervenes in a synergistic way in guidelines to direct and control the objectives of the Culture in the quality linked to productivity offers greater opportunities for the textile sector.

Productivity starts with efficiency and is looking for more performance with minimum resources. In the productivity is also the effectiveness whose purpose is to achieve the objectives proposed. The efficiency and the effectiveness result the effectiveness that is based on the productivity.

The rate of total productivity in the manufacturing industry in Ecuador as measured by the total production on total inputs which consists in CIIU3, paragraph 17 of the National Institute of Statistics and Census provides the standard of $\$ 2.0$ per unit of input. On the basis of the information the survey was calculated productivity from a mathematical approach that results of the productive system on the amount of resources used, concludes that the textile companies with certification have an index of 1.44 in productivity, while those who do not possess, the index is 1.20; therefore is required to socialize the new version for certification under the new requirements.

Keywords: System, quality management, productivity, textile.

\section{Introducción.}

El sistema de gestión de calidad son lineamientos que permiten administrar una organización con certificación así mismo busca evaluar el impacto en la productividad mediante el cumplimiento de los resultados esperados y la optimización de recursos utilizados en las empresas del sector textil. Es importante establecer criterios estandarizados en procesos de producción que permitan evidenciar la elaboración y evaluación de resultados. Por tanto, el desafío actual de las empresas es el desarrollo y permanencia dentro del mercado para la búsqueda de ventajas competitivas en el medio, una de estas es la implementación de la gestión de calidad. El conocer ¿qué impacto tiene la aplicación del sistema de gestión de calidad en la productividad de las empresas del sector textil de la provincia de Tungurahua?; crea la necesidad de identificar factores que determinen el costo-beneficio del sistema de gestión de calidad en las industrias textiles y por consiguiente estas oferten productos de calidad para mejorar la percepción del cliente y el sector diversifique las estrategias de gestión para ser productivas (Puerto, 2010). La industria textil es un sector diverso y juega un papel muy importante dentro de la manufactura a nivel nacional y local como segundo eje productivo analizado en el objetivo número 10 del Plan Nacional del Buen Vivir del Ecuador dado que es importante impulsar la transformación de la matriz productiva de acuerdo a lo establece el numeral 1, literal c., que manifiesta que: ... es importante consolidar la trasformación productiva de los sectores industriales y de manufactura con procesos de incorporación de valor agregado para fortaleces la innovación y capacidad socio productiva (Secretaría Nacional de Planificación y Desarrollo, 2015). Además, en el 2011 el Ministerio 
de Coordinación de la Producción, Empleo y Competitividad manifiesta que la manufactura textil se concentra fundamentalmente en la provincia de Chimborazo, Tungurahua y Cotopaxi. Así también en Tungurahua la manufactura generó hasta 2011, 448 plazas de empleo, un millón de dólares correspondientes a sueldos y salarios, y su producción bruta para la venta en 9,8 millones de dólares, esto nos hace entender la relevancia del sector y su aporte a la economía nacional.

Se trabajó desde la perspectiva constructivista como resultado de una actividad en donde se pretende resolver el problema de manera proactiva. El propósito es evidenciar si el sector textil aplica sistemas de gestión de calidad y elevar los niveles de productividad de las empresas para obtener ventajas competitivas y comparativas.

El tipo de investigación utilizada fue la descriptiva y como técnica la encuesta para la recolección de datos. La muestra de 320 empresas en base a la población de 1881 que están legalmente registradas con su respectivo nombre comercial en el Ministerio de Industrias y de la Productividad del Ecuador. Se identificó las empresas que poseen sistema de gestión de calidad y el impacto en la productividad, de esta forma los resultados señalaron que si existe relación entre el sistema de calidad y la productividad dentro de las empresas y concluimos que el uso del sistema de gestión de calidad es la herramienta de trabajo sinérgica de mucha utilidad dentro de las organizaciones del sector textil.

Como fundamento teórico se tomó a las normas ISO 9001 versión 2015, se publicaron el 23 de septiembre de 2015 y el periodo para que las empresas que tengan la certificación vigente ISO 9001:2008 es de tres años, pero es claro que llegar a cumplir con estos cambios conlleva esfuerzo y compromiso para las empresas y la dedicación con las que estas trabajen se verá reflejado es su productividad y competitividad (Escuela Europea de Excelencia, 2015). Las normas ISO buscan estandarizar los procesos y ayudan a prever desperdicios en producción textil para satisfacer las necesidades de la sociedad (ISO 9001, 2015). Implementar gestión de calidad es necesario para sobresalir ante la competencia y ofrece productos a bajo costo y con utilidad equilibrada (Cagnazzo, L., Taticchi, P. \& Fuiano, F., 2010). El objetivo principal del sistema de gestión de calidad es reducir sistemáticamente de la variabilidad de las características del producto con características de precisión y exactitud requerida por los clientes. Implementar programas de control de calidad en procesos productivos textiles permitirá efectuar análisis oportunos.

Un sistema de gestión de la calidad es un tema estratégico el cual debe ser tomado de la forma más clara y oportuna posible, los factores que tienen incidencia en este son: el entorno de la empresa, sus posibles cambios y a su vez los riesgos, los requerimientos variables, objetivos específicos de esta, los productos que realiza, los procesos que utiliza, el tamaño y su estructura (Bonilla, 2015). Estas son claves tanto para una decisión estratégica como para la utilización de un sistema de gestión de calidad y por así decirlo, es a lo que se refiere optar por un sistema de gestión basado en calidad (Gaviria y Dovale, 2014).

En la nueva versión ISO 9001:2015, encontramos siete principios de la gestión de la calidad como lo son el enfoque al cliente, liderazgo, compromiso y competencias de las personas, enfoque basado en procesos, mejora, toma de decisiones informadas, gestión de las relaciones (ISO 9001, 2015). 
Los beneficios de los sistemas de gestión de calidad radican en que están orientados a la mejora continua, son versátiles a los cambios del entorno, la toma de decisiones se basa en datos específicos que buscan evitar riesgos y se convierte en una ventaja competitiva para ser productiva a la organización (Carmona, Suárez, Calvo y Periáñez, 2015).

La productividad en términos aritméticos se la puede considerar como el cociente del sistema productivo y la cantidad de recursos utilizados, pero en la práctica este término hace referencia a una variable que identifica que tan cerca o lejos nos encontramos del objetivo primordial del sistema (Salazar, 2016).

La productividad para las empresas en el 2010 se ha centrado tanto en la definición e implementación de procesos de mejora en industrias a nivel mundial, a través de impulsos estratégicos o de mejora de procesos mismos que se debe buscar para el desarrollo del sector productivo de la provincia (Fundación Telefónica, 2011). La mejora en la productividad jamás ha estado más ligada con la calidad como se encuentra en la actualidad (Fernández, 2010). Entre una de las formas básicas para mejorar la productividad están, incrementar resultados sin incrementar los insumos (Hernández y Palafox, 2012); según Forero (2014) hay dos tipos de factores que incurren en la productividad y que pueden ayudar a mejorar la productividad, estos son los factores internos como tecnologia, talento humano, investigacion y desarrollo, entre otros y los externos como: factores demograficos, culturales, políticos, otros que estan en el entorno de la organización.

Los indicadores se basan en dos principios donde expresan que lo que no es medible no es gerenciable y el control se ejerce a partir de hechos y datos historicos (Sistemas para la gestión de la información y las comunicaciones estratégicas., 2011). Los indicadores de gestión dentro de un sistema de producción es muy importante a razón de que, gracias a estos se puede llevar un control de los ciclos y de los procesos del sistema (Salazar, 2016).

Los indicadores pueden variar acorde y según los requerimientos de la organización en donde se los emplee, los indicadores tradicionales se los utilizaba en su gran parte para obtener resultados que evaluaran el desempeño del área de ventas, producción, competitividad en el mercado, en la actualidad conocemos que para poder determinar indicadores de productividad se requiere definir un objetivo el mismo que justificara y este será en términos de calidad, cantidad y tiempo (Camejo, 2012).

Espinoza y Sorhegui (2016), el análisis de los niveles de productividad en el sector textil ecuatoriano de acuerdo a los argumentos citados por Bertola \& Ocampo (2013) en Espinoza y Sorhegui se requiere transformar la política de exportación orientada a fomentar actividades productivas con ventajas comparativas y competitivas dinámicas, que impulsen encadenamientos productivos, innovación tecnológica y valor agregado para elevar los niveles de productividad, competitividad sistémica y reactiven la demanda interna en la industria textil. Pérez (2016) propone el análisis comparativo (tabla 2) de la estructura entre las versiones de las normas ISO 9001: 2008 a la 2015, para el sector ecuatoriano en donde 
se evidencia cambios estructurales en el sistema de gestión de la calidad y se relacionan en el contexto de la organización, liderazgo y planificación en relación a la responsabilidad de la dirección, apoyo a la gestión de los recursos, operacionalización del producto y evaluación del desempeño con mejora en la medición y análisis.

\section{Marco teórico referencial.}

La investigación descriptiva es una forma de estudio donde determinamos características de un grupo se lo puede considerar que posee una forma rígida (Rodríguez, Pierdant y Rodríguez, 2011); es también aquella rama de la estadística que se encarga mediante la aplicación de métodos y técnicas de obtener, organizar, presentar y describir los datos (Namakforoosh, 2010). Por tanto, se utilizó este tipo de investigación para determinar el uso del sistema de gestión de calidad, enfocadas a la productividad y métodos de control para definir los niveles de efectividad en la producción y facilitar la estandarización pertinente (Koontz, Weihrich, \& Cannice, 2012). Buscamos encontrar experiencias y percepciones del grupo de empresarios textiles y la información obtenida de la investigación bibliográfica la cual generó los conocimientos para sustentar la información documental relacionada al tema. En la investigación de campo por la cercanía de las empresas en la provincia se buscó obtener información dentro de las organizaciones textiles de la provincia y así detectar si la calidad afecta a la productividad en este sector. La muestra para el estudio es de 320 empresas en base a la población de 1881 que están legalmente registradas con su respectivo nombre comercial en el Ministerio de Industrias y de la Productividad del Ecuador (Bernal, 2010). Se utilizó la encuesta como herramienta de investigación para evidenciar datos y así generaremos resultados a base de la misma (Rodríguez, Pierdant y Rodríguez, 2011). Para validar la encuesta y verificar como instrumento fiable y sustentar el nivel de relación entre los ítems se comprobó mediante el alfa de Cronbach como coeficiente superior a 0,7 (Bojórquez, J., López, L., Hernández, M. y Jiménez, E., 2013).

\section{Resultados.}

En los resultados se evidenció que el $85 \%$ de las empresas de la provincia de Tungurahua no poseen sistema de gestión de calidad y apenas el $15 \%$ si lo tiene, es decir que pocos son los que poseen certificación ISO 9001:2008 o la versión 2015 debido a la escasa motivación al sector por parte de las autoridades, así también por costes de inversión que muchas empresas pequeñas no poseen (Tabla 1).

Tabla 1: Sistema de gestión de calidad 


\begin{tabular}{cccccc}
\hline & & Frecuencia & Porcentaje & Porcentaje válido & $\begin{array}{c}\text { Porcentaje } \\
\text { acumulado }\end{array}$ \\
\hline Válidos & No & 272 & 85,0 & 85,0 & 85,0 \\
& Si & 48 & 15,0 & 15,0 & 100,0 \\
& Total & $\mathbf{3 2 0}$ & $\mathbf{1 0 0 , 0}$ & $\mathbf{1 0 0 , 0}$
\end{tabular}

Fuente: Encuestas a empresas del sector textil provincia de Tungurahua, 2016.

Elaborado por: Evelyn Cepeda.

El 78.4\% considera que es muy importante (Tabla 2) para la empresa el sistema de gestión de calidad y la productividad, apenas el $21.6 \%$ considera que es poca la importancia de estos. Esto pese a que más de la mitad señaló que no cuentan con un sistema de gestión de calidad, lo cual muestra que estas podrían optar por un sistema de gestión de calidad para futuro

Tabla 2: Importancia de los sistemas de gestión de calidad y la productividad en la empresa

\begin{tabular}{|c|c|c|c|c|c|}
\hline & & Frecuencia & Porcentaje & Porcentaje válido & $\begin{array}{l}\text { Porcentaje } \\
\text { acumulado }\end{array}$ \\
\hline \multirow[t]{3}{*}{ Válidos } & Poco & 69 & 21,6 & 21,6 & 21,6 \\
\hline & Mucho & 251 & 78,4 & 78,4 & 100,0 \\
\hline & Total & 320 & 100,0 & 100,0 & \\
\hline
\end{tabular}

Fuente: Encuestas a empresas del sector textil provincia de Tungurahua, 2016.

Elaborado por: Evelyn Cepeda.

Para la debida medición comparativa del impacto en la productividad, se realizó el cálculo matemático mediante la aplicación de la fórmula 01 de productividad (Instituto Nacional de Estadística y Geografía de México, 2016); mediante el uso de los datos correspondientes (Tabla 3).

Para calcular, remplazamos valores en la fórmula 01: 


$$
\text { Productividad }=\frac{\text { Sistema Productivo }}{\text { recursos utilizados }}
$$

(Fórmula 01: Productividad)

Empresas que si tienen sistema de gestión de calidad:

$$
\begin{gathered}
\text { Productividad }^{1}=\frac{1938500}{1345000} \\
\text { Productividad }^{1}=1,4412
\end{gathered}
$$

Para calcular de las empresas que no tiene sistema de gestión de calidad:

$$
\begin{aligned}
\text { Productividad }^{2} & =\frac{582500}{483500} \\
\text { Productividad }^{2} & =1,2047
\end{aligned}
$$

Aquí podemos encontrar la diferencia en el cálculo matemático de la productividad de los dos casos, en las empresas que si tienen sistema de gestión de calidad, encontramos el 1.44 mientras que, en las que no lo tienen es de 1.20, lo cual indica que al trabajar con un sistema de gestión de calidad dentro de la empresa esta es mayormente productiva.

Tabla 3: Suma de totales según clasificación.

\begin{tabular}{cccc}
\hline Etiquetas de fila & $\begin{array}{c}\text { Suma de } \\
\text { Costo }\end{array}$ & $\begin{array}{c}\text { Suma de } \\
\text { Precio }\end{array}$ & $\begin{array}{c}\text { Suma de } \\
\text { Ventas }\end{array}$ \\
\hline No & 483500 & 569200 & 582500 \\
Si & 1345000 & 1530500 & 1938500 \\
Total general & $\mathbf{1 8 2 8 5 0 0}$ & $\mathbf{2 0 9 9 7 0 0}$ & $\mathbf{2 5 2 1 0 0 0}$ \\
\hline
\end{tabular}

Fuente: Encuestas a empresas del sector textil provincia de Tungurahua, 2016. Elaborado por: Evelyn Cepeda.

Para poder presentar los datos encontrados como fiables se aplicó el método estadístico de chi-cuadrado el cual se expone con los siguientes datos: (Tabla 4).

\section{Nivel de significancia:}

En la investigación se trabajó con un nivel de significancia del 5\%, que indica que existe una probabilidad del 95\% de que la hipótesis nula sea verdadera.

Tabla 4: Tabla de contingencia

$\begin{array}{lcc}\text { Tabla de contingencia } & \text { ¿Cuenta la empresa } & \text { Tota } \\ \text { con un sistema de } & 1 \\ \text { gestión de calidad? } & \end{array}$


ISSN 2602-8506

Vol. 2 / No.1/ pág. 25-37. Enero-Marzo/2018

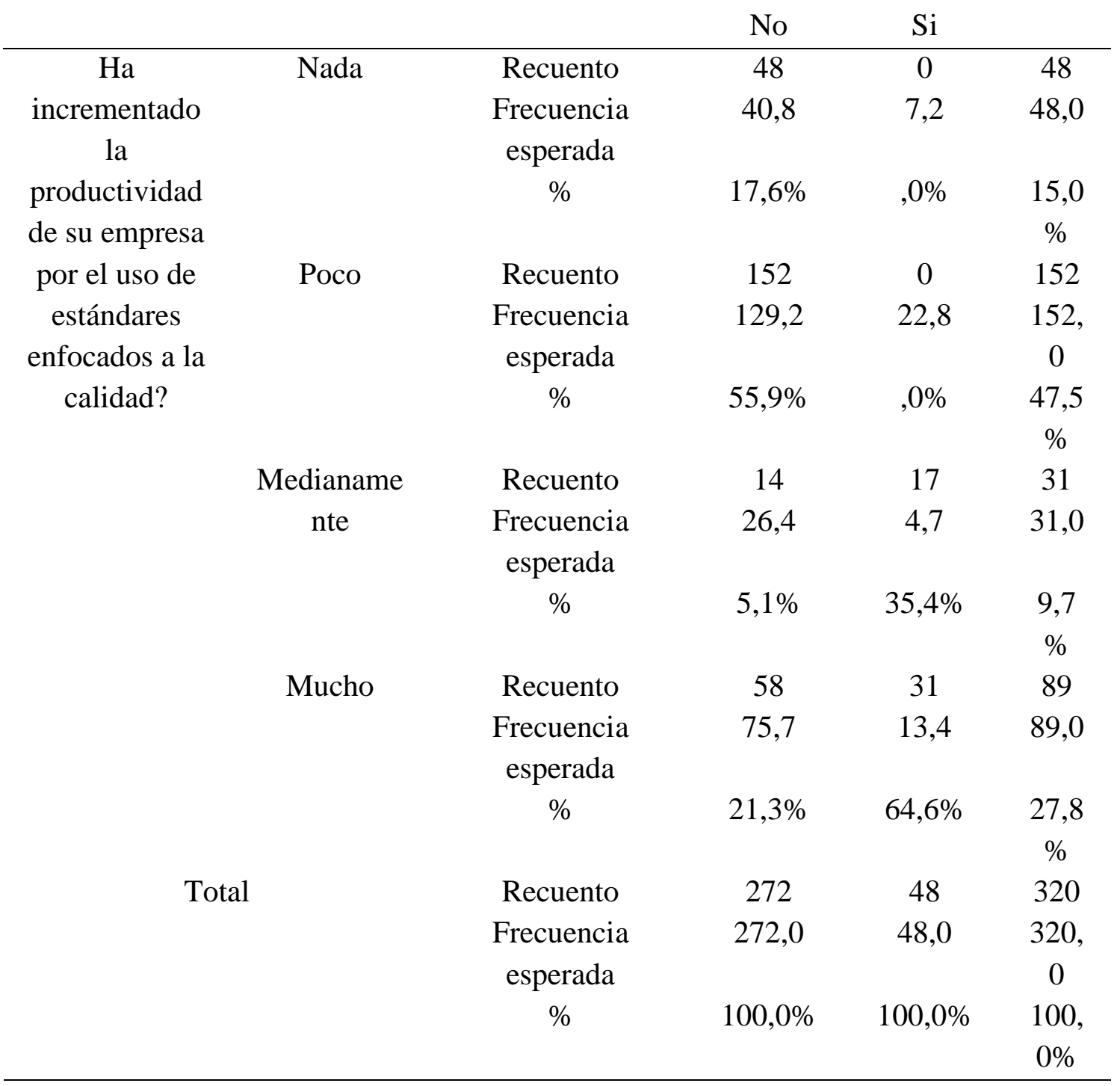

Fuente: Encuestas a empresas del sector textil provincia de Tungurahua, 2016.

Elaborado por: Evelyn Cepeda.

Tabla 5: Pruebas de chi-cuadrado

\begin{tabular}{lll}
\hline Valor & gl & $\begin{array}{l}\text { Sig. asintótica } \\
\text { (bilateral) }\end{array}$
\end{tabular}

$\begin{array}{llll}\text { Chi-cuadrado de Pearson } & 101,336^{\mathrm{a}} & 3 & , 000\end{array}$

$\begin{array}{llll}\text { Razón de verosimilitudes } & 112,791 & 3 & , 000\end{array}$ 
$\mathrm{N}$ de casos válidos

320

Nota: a. 1 casillas $(12,5 \%)$ tienen una frecuencia esperada inferior a

5. La frecuencia mínima esperada es 4,65.

Fuente: Encuestas a empresas del sector textil provincia de Tungurahua, 2016.

Elaborado por: Evelyn Cepeda.

\section{Decisión estadística}

Luego de realizado la prueba del estadístico, se encontró que nos da 0.00 el cual es menor a 0.05 del nivel de significancia, lo que permite rechazar la hipótesis nula y aceptar la hipótesis alternativa, donde se afirma que, el sistema de gestión de calidad si incide en la productividad dentro de las empresas de la provincia de Tungurahua.

Se pudo encontrar que el uso del sistema de gestión de calidad es una herramienta de trabajo de mucha utilidad dentro de las empresas y especialmente dentro del sector textil (Fraiz, Àlvarez y De la Cruz, 2012), pero dentro de la provincia se evidencia que son muchas las empresas que aún no persiguen una visión enfocada a la calidad; pero, éstas son conscientes de la importancia de la gestión de calidad dentro del sector.

Cuando miramos la productividad de las empresas con sistema de gestión de calidad se puede identificar que son mayormente productivas que las que no poseen sistema de gestión, pero el capital de trabajo de muchas empresas busca generar calidad de forma empírica a causa de requerir una inversión para su implementación y muchas no lo tienen.

\section{Conclusiones.}

El sistema de gestión de calidad beneficia aspectos como: financieros, operativos, comerciales y costos. Este sistema internamente relaciona aspectos de: satisfacción y seguridad, ausentismo, salarios, fiabilidad de las operaciones, entregas oportunas, cumplimiento en pedidos, reducción de errores y rotación de existencias; en lo externo, asocia a: satisfacción de clientes, quejas y reclamaciones, compras, cuota de mercado, rendimiento de ventas y activos. La productividad desde un enfoque matemático es el resultado del sistema productivo sobre la cantidad de recursos utilizados. La productividad, inicia con la eficiencia y busca mayor rendimiento con el mínimo de recursos además en la productividad está inmersa la efectividad cuyo propósito es conseguir los objetivos propuestos. La eficiencia y efectividad da como resultado la eficacia que es la base de la productividad. Se identificó a través de la investigación descriptiva, en base a la productividad total de la industria manufacturera ecuatoriana las empresas que poseen certificación de calidad tiene un índice de 1,44 en productividad mientras que las que no poseen, el índice es de 1.20.

Los resultados obtenidos en la investigación permitieron concluir que las empresas que tienen sistema de gestión de calidad tiene un índice de productividad del 1.44 mientras que 
las que no poseen la calificación obtuvieron un índice de 1.20; es decir que se pudo notar que si existe diferencia al relacionar el sistema de gestión de calidad y la productividad. La prueba estadística evidenció que si tienen incidencia el sistema de gestión de calidad en la productividad de las empresas del sector textil de la provincia de Tungurahua.

Sugerimos a las empresas del sector textil implementen el sistema de gestión de calidad en base a las normas vigentes, puesto que es un factores que buscan incrementar la productividad.

La capacitación al sector textil en temas de calidad y productividad serán ejes en beneficio propio donde el costo - beneficio sea atractivo para elevar niveles de eficiencia, eficacia, efectividad y obtener ventajas competitivas y comparativas con la competencia.

\section{Referencias}

Bernal, C. (2010). Metodología de la investigación: administración, economía, humanidades y ciencias sociales. Colombia: Pearson.

Bojórquez, J., López, L., Hernández, M. y Jiménez, E. (2013). Utilización del alfa de cronbach para validar la confiabilidad de un instrumento de medición de satisfacción del estudiante en el uso de software minitab. Eleventh Latin American and Caribbean conference for engineering and technoloy (págs. 23-29). Cancúm,México: LACCEI.

Bonilla, E. (2015). La gestión de la calidad y su relación con los costos de desechos y desperdicios en las mypes de la confección textil. Universidad de Lima: Portal de revistas Ulima, 33, 1-14.

Cagnazzo, L., Taticchi, P. \& Fuiano, F. (2010). Benefits, barriers and pitfalls coming from the ISO 9000 implementation: The impact on business performances. WSEAS Transactions on Business and Economics, 311-321

Camejo, J. (28 de noviembre de 2012). Gestiopolis. Recuperado de Administración: http://www.gestiopolis.com/indicadores-de-gestion-que-son-y-por-que-usarlos/

Carmona, M., Suárez, E., Calvo, A. y Periáñez, R. (2015). Quality management systems: A study in companies of southern Spain and northern Morocco. Revista Dialnet: European Research on Management and Business Economics, 8-16.

Espinoza, C. (2016). Análisis del sector textil ecuatoriano 2009- 2013. Revista Científica Ecociencia, 1-23.

Fernández, R. (2010). La mejora de la productividad en la pequeña y mediana empresa. España: Club Universitario-ECU.

Forero, C. (2014). El sistema de gestión de calidad como herramienta fundamental para lograr competitividad. Universidad Militar Nueva Granada, 1-11.

Fraiz, J., Àlvarez, J. y De la Cruz, M. (2012). Motivaciones para implementar un sistema de gestión de la calidad: Análisis empírico en el sector turístico español. Revista de Cultura y turismo, 40-68.

Hernandez, S. y Palafox, G. (2012). Administración: Teoría, proceso, áreas funcionales y estrategias para la competitividad. México: McGraw Hill. 
Instituto Nacional de Estadística y Geografía. (8 de noviembre de 2016). Instituto Nacional de Estadistica y Geografía. Recuperado de INEGI: http://www.inegi.org.mx/default.aspx

ISO 9001. (2015). Requisitos para los Sistemas de Gestión de la Calidad. Interpretación libre ISO/DIs 9001:2015, 33.

Koontz, H., Weihrich, H. \& Cannice, M. (2012). Administración: Una perspectiva global y empresarial. México: McGraw-Hill/ Interamericana Editores S.A.

Ministerio de Coordinación de la Producción, Empleo y Competitividad. (2011). Agendas para la Transformacion Productiva Territorial Provincia de Tungurahua. Ambato.

Ministerio de Industrias y de la Productividad. (2016). Empresas textiles de Tungurahua. Ambato, Tungurahua, Ecuador.

Namakforoosh, M. (2010). Metodología de la investigación. México D.F.: Editorial Limusa .

Perez, Y. (2016). Transito de la ISO 9001: 2008 a la 2015, una necesidad del sector empresarial ecuatoriano. Revista Valor Agregado. 109-132.

Puerto, P. (2010). Globalization and entrepreneurial growth through internationalization strategies. Revista Cientifica Pensamiento y Gestión, 28, 247-248.

Rodríguez, J., Pierdant, A. y Rodríguez, E. (2011). Estadistica para administración. Mexico: Grupo Patria.

Salazar, B. (2016). Ingeniería Industrial. Recuperado de Ingeniería Industrial online: www.ingenieriaindustrialonline.com

Secretaría Nacional de Planificación y Desarrollo. (2015). Agenda Zonal: Zona 3- Centro. Ecuador: Senplades.

Sistemas para la gestión de la información y las comunicaciones estratégicas. (2 de noviembre de 2011). Recuperado de Los indicadores de gestión como elemento de medición en las estratégias de comunicaciones y marketing: https://sisgecom.com/2011/11/02/los-indicadores-de-gestion-como-elemento-demedicion-en-las-estrategias-de-comunicaciones-y-marketing/

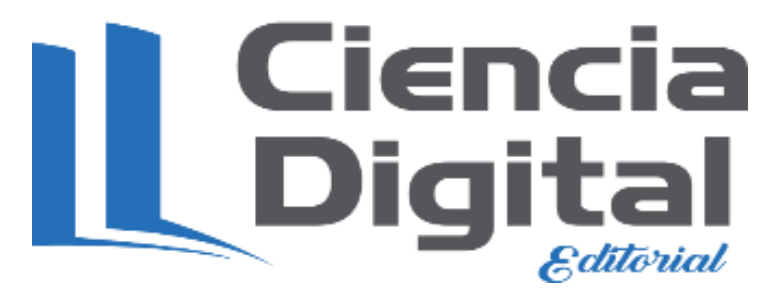




\section{Para citar el artículo indexado.}

Ramos E.,Velasteguí L., Cepeda, E. \& Carrasco T.(2018). Sistema de gestión de calidad y su impacto en la productividad del sector textil. Revista electrónica VisionarioDigital1(1), 25-37.

\section{【Liencia}

El artículo que se publica es de exclusiva responsabilidad de los autores y no necesariamente reflejan el pensamiento de la Revista Ciencia Digital.

El articulo queda en propiedad de la revista y, por tanto, su publicación parcial y/o total en otro medio tiene que ser autorizado por el director de la Revista Ciencia Digital. 